\title{
Compact zeroth-order resonator (ZOR) antennas
}

\author{
Jae-Gon Lee ${ }^{1}$ and Jeong-Hae Lee ${ }^{2, *}$ \\ ${ }^{1}$ Metamaterial Electronic Device Research Center, Hongik University, Seoul 04066, Korea \\ ${ }^{2}$ School of Electronic and Electrical Engineering, Hongik University, Seoul 04066, Korea
}

Received: 17 September 2018 / Accepted: 2 January 2019

\begin{abstract}
In this paper, we introduce and review the zeroth-order resonator (ZOR) antennas with outstanding characteristics including various applications that have been researched so far. Since the zeroth-order resonance frequency is independent of a physical length of antenna, the ZOR antenna can theoretically be designed quite small and have a possibility to apply to considerably lots of applications. First, we have presented the ZOR antennas implemented by double-negative (DNG), epsilon-negative (ENG), and mu-negative (MNG) transmission lines. Then, the research related on extremely small, wide beamwidth, wideband, and circularly polarized (CP) ZOR antennas have been continuously carried out. Based on a series of these studies, the ZOR antennas were utilized for various applications such as a wireless power transfer (WPT), a compact controlled reception pattern antenna (CRPA), a penta-band mobile antenna, and a wide steering array antenna.
\end{abstract}

Keywords: Double-negative (DNG) / epsilon-negative (ENG) / mu-negative (MNG) / zeroth-order resonator / circularly polarized (CP) / wide beamwidth / wideband

\section{Introduction}

Double-negative (DNG) materials, first introduced and analyzed theoretically by V.G. Veselago in 1968 [1], have received considerable attention since their unique properties were experimentally demonstrated in 2001 [2]. However, the artificial DNG materials composed of conducting split ring resonators (SRRs) and periodic thin wires are impractical because of their narrow bandwidth and lossy characteristics. In [3,4], a transmission line approach of DNG materials, which is composed of the combination of series capacitance and shunt inductance, was introduced for the radio frequency $(\mathrm{RF})$ devices. The DNG transmission line provides an inherent parasitic DPS property because it contains the same components utilized in a conventional DPS transmission line. In addition, the DNG transmission line has a low level of loss and a broad bandwidth owing to the different type of dispersion relation comparing to the SRR-wire structure so that it has been utilized to design $\mathrm{RF}$ devices with the unique properties [5-12]. A lot of research using the DNG transmission line has been done in the radiated wave applications such as resonator-type antennas and leaky-wave antennas as well as in the guided wave applications such as RF devices.

In the paper, the various zeroth-order resonator (ZOR) antennas are explained and reviewed. The DNG transmission

\footnotetext{
* e-mail: jeonglee@hongik.ac.kr
}

line with an inherent parasitic DPS property has the unique feature of an infinite wavelength wave (zero propagation constant) at a discrete frequency at the boundary of the DNG and DPS bands. Using the infinite wavelength property of the DNG transmission line, the ZOR [13] and ZOR antennas $[14,15]$ have been reported. In [15], the horizontal magnetic loop current, which gives rise to omnidirectional radiation, has been realized by the ZOR antenna using the mushroom structure. Then, the epsilon-zero resonance (EZR) and muzero resonance (MZR) antennas were proposed sequentially $[16,17]$. The EZR antenna consists of series and shunt inductances and can be obtained by open-ended boundary condition. Similarly, the MZR antenna consists of series and shunt capacitances and can be obtained by short-ended boundary condition. Moreover, there are various studies to improve the performance of the ZOR antenna such as an electrically small, wideband, and wide beamwidth [18-20]. The circularly polarized (CP) ZOR antenna was also proposed and developed [21,22]. The results of this fundamental research have been applied to various practical RF front-ends [23-26]. In Section 2, the meta-structured transmission lines for DPS, DNG, epsilon-negative (ENG), and mu-negative (MNG) materials and their dispersion diagrams are described. Then, the ZOR antennas including EZR and MZR antennas, and the improved variety of ZOR antennas including CP ZOR antenna are explained and discussed in Section 3. The several kinds of applications using the ZOR antenna and conclusion are presented in Sections 4 and 5 , respectively. 


\section{Meta-structured transmission lines and their resonance frequencies}

Figure 1 shows differential equivalent circuits of lossless ( $R=0$ and $G=0$ ) DPS, DNG, ENG, and MNG transmission lines. The DPS transmission line can be represented as the combination of a per-unit length series inductance $\left(L_{R}^{\prime}\right)$ and shunt capacitance $\left(C_{R}^{\prime}\right)$ in terms of an infinitesimal component (in $H / m$ and $F / m$ ). Since the DNG transmission line has an inherent parasitic DPS property, it can be represented as the combination of a perunit length series inductance $\left(L_{R}^{\prime}\right)$, shunt capacitance $\left(C^{\prime}{ }_{R}\right)$, a time-unit length series capacitance $\left(C^{\prime}{ }_{L}\right)$, and shunt inductance $\left(L_{L}^{\prime}\right)$ in terms of an infinitesimal component (in $H / m, F / m, F \cdot m$, and $H \cdot m$ ). Also, the ENG and MNG transmission lines are represented by adding a time-unit length shunt inductance $\left(L^{\prime}{ }_{L}\right)$ and series capacitance $\left(C^{\prime}{ }_{L}\right)$ in terms of an infinitesimal component (in $H \cdot m$ and $F \cdot m$ ) to the equivalent circuit of the DPS

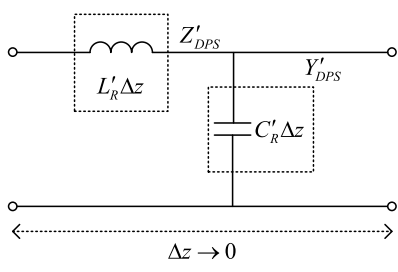

(a)

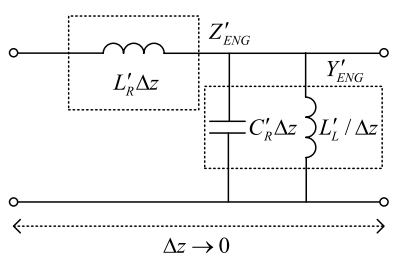

(c)

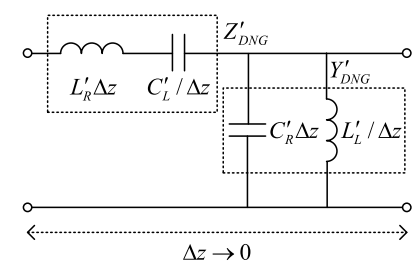

(b)

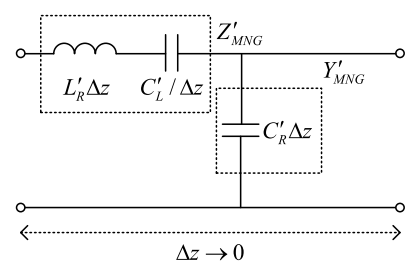

(d)
Fig. 1. Infinitesimal equivalent circuits of lossless (a) DPS, (b) DNG, (c) ENG, and (d) MNG transmission lines. transmission line, respectively. The effective permittivity and permeability of the four-transmission lines based on expression of $\beta=\omega \sqrt{\mu \varepsilon}$ can be obtained as

$$
\begin{aligned}
& \varepsilon_{D P S}=C_{R}^{\prime}, \quad \mu_{D P S}=L_{R}^{\prime} \\
& \varepsilon_{D N G}=C_{R}^{\prime}-\frac{1}{\omega^{2} L_{L}^{\prime}}, \quad \mu_{D N G}=L_{R}^{\prime}-\frac{1}{\omega^{2} C^{\prime}{ }_{L}} \\
& \varepsilon_{E N G}=C_{R}^{\prime}-\frac{1}{\omega^{2} L_{L}^{\prime}}, \quad \mu_{E N G}=L_{R}^{\prime} \\
& \varepsilon_{M N G}=C_{R}^{\prime}, \quad \mu_{M N G}=L_{R}^{\prime}-\frac{1}{\omega^{2} C_{L}^{\prime}} .
\end{aligned}
$$

A propagation constant of DNG, ENG, and MNG transmission lines can also be expressed by applying the Bloch and Floquet theory to the unit cell as follows.

$$
\begin{aligned}
& \beta_{D N G} d=\cos ^{-1}\left\{1-\frac{1}{2}\left[\frac{\omega_{L}^{2}}{\omega^{2}}+\frac{\omega^{2}}{\omega_{R}^{2}}-\left(\frac{\omega_{E}^{2}}{\omega_{R}^{2}}+\frac{\omega_{M}^{2}}{\omega_{R}^{2}}\right)\right]\right\} \\
& \beta_{E N G} d=\cos ^{-1}\left\{1-\frac{1}{2}\left(\frac{\omega^{2}-\omega_{E}^{2}}{\omega_{R}^{2}}\right)\right\} \\
& \beta_{M N G} d=\cos ^{-1}\left\{1-\frac{1}{2}\left(\frac{\omega^{2}-\omega_{M}^{2}}{\omega_{R}^{2}}\right)\right\}
\end{aligned}
$$

where $\omega_{R}=1 / \sqrt{L_{R} C_{R}}, \omega_{L}=1 / \sqrt{L_{L} C_{L}}, \omega_{M}=1 / \sqrt{L_{R} C_{L}}$, and $\omega_{E}=1 / \sqrt{L_{L} C_{R}} \cdot \beta$ and $d$ are a phase constant for Bloch waves and a length of the unit cell, respectively. $C_{R}, L_{R}, C_{L}$, and $L_{L}$ are the shunt capacitance, series inductance, series capacitance, and shunt inductance in terms of the real lumped component (in $F$ and $H$ ), respectively. From equation (2), the dispersion diagrams of the three artificial meta-structured transmission lines are obtained and those of the DNG, ENG, and MNG transmission lines are compared with the ZOR frequencies of each transmission line, as shown in Figure 2. The equivalent circuit element values of the dispersion diagram are $C_{R}=1 \mathrm{pF}, L_{R}=1 \mathrm{nH}$, $C_{L}=2 \mathrm{pF}$, and $L_{L}=1 \mathrm{nH}$, respectively. In Figure 2, epsilon-zero $\left(f_{E}\right)$ and mu-zero $\left(f_{M}\right)$ are $5.03 \mathrm{GHz}$ and $3.56 \mathrm{GHz}$, respectively. The propagation constants of the

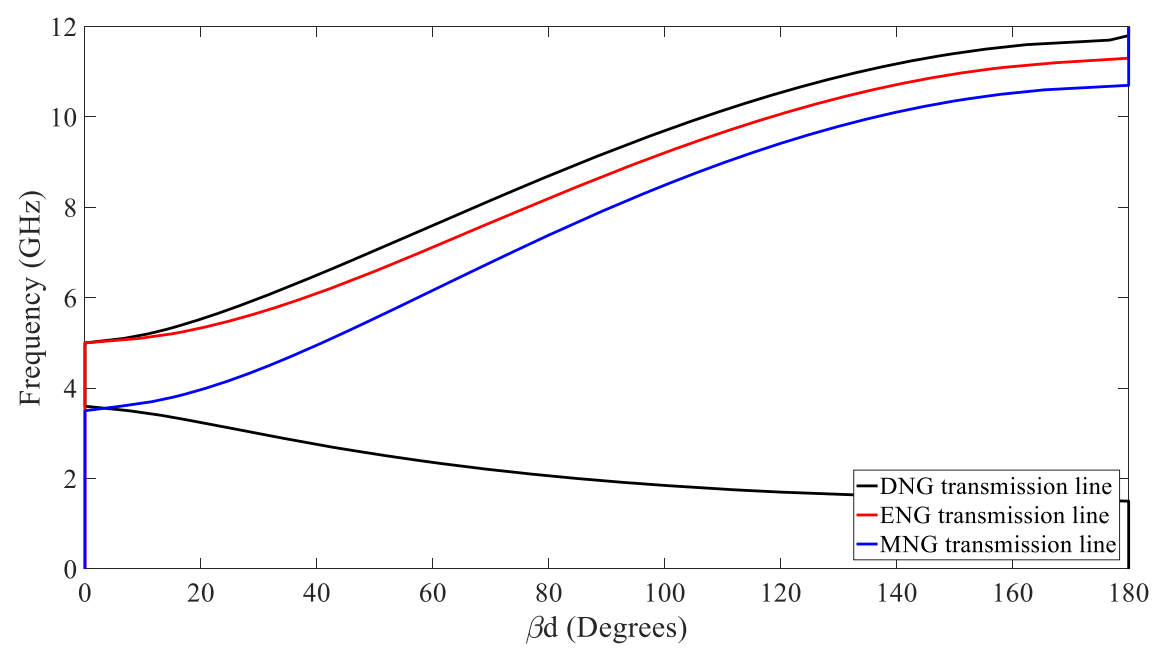

Fig. 2. Dispersion curves of lossless (a) DNG, (b) ENG, and (c) MNG transmission lines. 


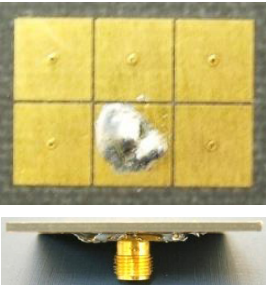

(a)

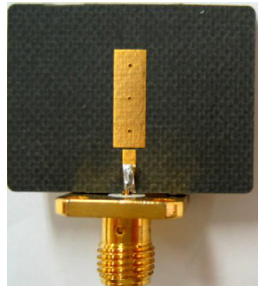

(b)

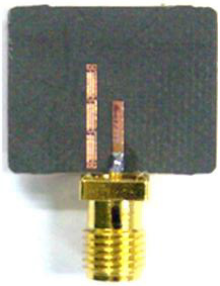

(c)
Fig. 3. ZOR antennas using the (a) DNG, (b) ENG, and (c) MNG transmission lines [15-17].

DNG transmission line are negative, zero, and positive values, while the ENG and MNG transmission lines support zero and positive propagation constants.

The resonance of the meta-structured transmission line for resonance modes $(n)$ can be obtained by the following condition

$$
\begin{aligned}
& \beta_{n}=\frac{n \pi d}{l}=\frac{n \pi}{N} \\
& \left\{\begin{array}{l}
D N G: n=0, \pm 1, \pm 2, \ldots, \pm(N-1) \\
E N G: n=0,1,2, \ldots,(N-1) \\
M N G: n=0,1,2, \ldots,(N-1)
\end{array}\right.
\end{aligned}
$$

where $N(=l / d)$ and $l$ are the number of unit cell and total length of resonator, respectively. To calculate the theoretical ZOR frequency of the meta-structured transmission lines, the input impedances based on the open- and shortended boundary conditions are calculated by (4) [13].

$$
\begin{aligned}
& \text { Open }- \text { ended boundary condition : } \\
& \begin{aligned}
Z_{i n} & =-\left.j \cot \beta l\right|_{\beta=0}=-j Z_{0} \frac{1}{\beta l} \\
& =-j \sqrt{\frac{Z^{\prime}}{Y^{\prime}}}\left(\frac{1}{-j \sqrt{Z^{\prime} Y^{\prime}}}\right) \frac{1}{l}=\frac{1}{N Y}
\end{aligned}
\end{aligned}
$$

Short - ended boundary condition :

$$
\begin{aligned}
Z_{i n} & =\left.j \tan \beta l\right|_{\beta=0}=j Z_{0} \beta l \\
& =j \sqrt{\frac{Z^{\prime}}{Y^{\prime}}}\left(-j \sqrt{Z^{\prime} Y^{\prime}}\right) l=N Z
\end{aligned}
$$

The EZR and MZR frequencies can be obtained by the open- and short-ended boundary condition, respectively. Both boundary conditions are available to obtain the ZOR frequency of the DNG transmission line. The EZR frequency is given as $1 / \sqrt{L_{L} C_{R}}$, because $Z_{i n}$ can be expressed by the impedance of the $L C$ anti-resonant tank. Similarly, the MZR frequency is given as $1 / \sqrt{L_{R} C_{L}}$, because $Z_{\text {in }}$ can be expressed by the impedance of the $L C$ resonant tank.

\section{Compact ZOR antennas}

To realize the DNG transmission line, the mushroom structure composed of combination of a rectangular patch with a series gap and grounded via hole is employed. The DNG transmission line using the mushroom structure has left-handed characteristic by series capacitance and shunt inductance. Moreover, the ENG and MNG transmission lines are realized with only grounded via hole and only series gap, respectively. Figure 3 shows the ZOR antennas using the DNG, ENG, and MNG transmission lines. In both cases of DNG and ENG ZOR antennas, each ZOR has uniform electric field at zeroth-order resonant frequency because of infinite wavelength and open-ended boundary condition. Thus, the radiation patterns of the ZOR antennas are omnidirectional, as shown in Figure $4 \mathrm{a}$. Figure $4 \mathrm{~b}$ shows the simulated and measured radiation pattern of MZR antenna. Since the radiation mechanism of the MZR antenna is the same as that of a conventional small loop antenna, the MZR antenna radiates to broadside.

To design electrically small MZR antenna, the spiral structure is employed, because the MZR frequency is given as $1 / \sqrt{L_{R} C_{L}}$ and $L_{R}$ is an inherent series inductance. The spiral line on top plate is connected to spiral line on bottom through the centered via. To improve the radiation efficiency, the MZR antenna with the same current direction on top and bottom plate is designed, as shown in Figure 5 [18]. As a result, the MZR antenna using multi-layered spiral structure $(k r=0.182)$ has a measured $3 \mathrm{~dB}$ fractional bandwidth of $0.846 \%$ and radiation efficiency of $18.66 \%$. Meanwhile, a wideband folded ZOR antenna and a hybrid ZOR antenna with a broad E-plane beamwidth are studied to improve the performance of the ZOR antenna. The wideband ZOR antenna is achieved by the folded mushroom structure operated over two modes (ZOR and $\mathrm{TM}_{010}$ modes), as shown in Figure 6. The $-10 \mathrm{~dB}$ fractional bandwidth is measured as $68.3 \%$. In addition, the hybrid ZOR antenna with a broad E-plane beamwidth is designed by combining $\mathrm{TM}_{010}$ and $\mathrm{ZOR}$ modes. To generate the $\mathrm{TM}_{010}$ mode and the ZOR mode, simultaneously, the mushroom structure is inserted in a rectangular patch antenna and a single feed is employed between two radiators, as shown in Figure 7 . By the omnidirectional radiation pattern of the $\mathrm{ZOR}$ mode, the E-plane beamwidth of a $\mathrm{TM}_{010}$ mode can easily be broadened. The E-plane HPBW of the antenna is measured to be $115^{\circ}$ and it is about $50 \%$ broader than that of the conventional rectangular patch antenna.

We have researched CP ZOR antennas as well as linearly polarized (LP) ZOR antennas. Among various CP ZOR antennas, the omnidirectional CP EZR antenna is described below. The antenna is based on the ZOR mode of the ENG transmission line to obtain a vertical polarization and an omnidirectional radiation pattern. Also, the horizontal polarization is obtained by the curved branches, as shown in Figure 8 . The $90^{\circ}$ phase difference between two orthogonal polarizations is inherently provided by the ZOR. Therefore, the antenna has an omnidirectional CP radiation pattern in the azimuthal plane. In addition, this antenna is of planar type and simply designed without a dual feeding structure and $90^{\circ}$ phase shifter. The measured average axial ratio and left-hand $(\mathrm{LH}) \mathrm{CP}$ gain are $2.03 \mathrm{~dB}$ and $-0.40 \mathrm{dBic}$, respectively, in the azimuthal plane, as shown in Figure 9. 


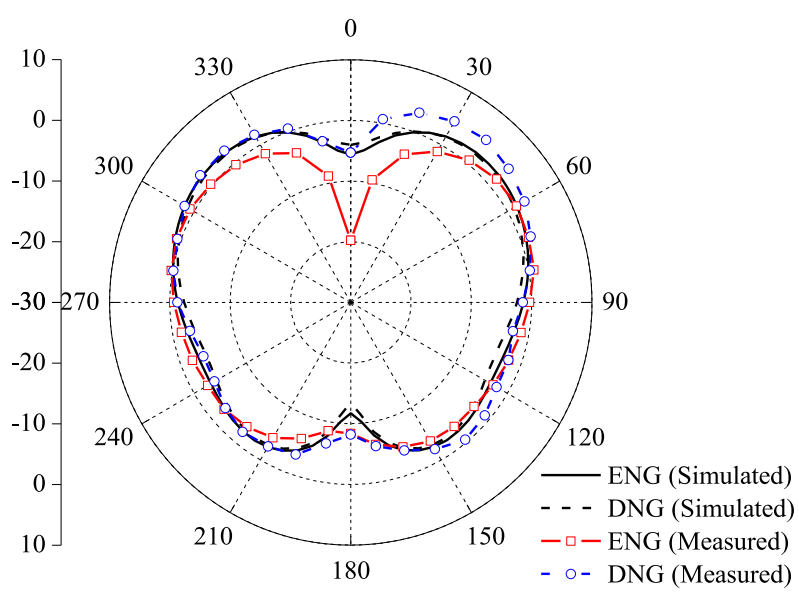

(a)

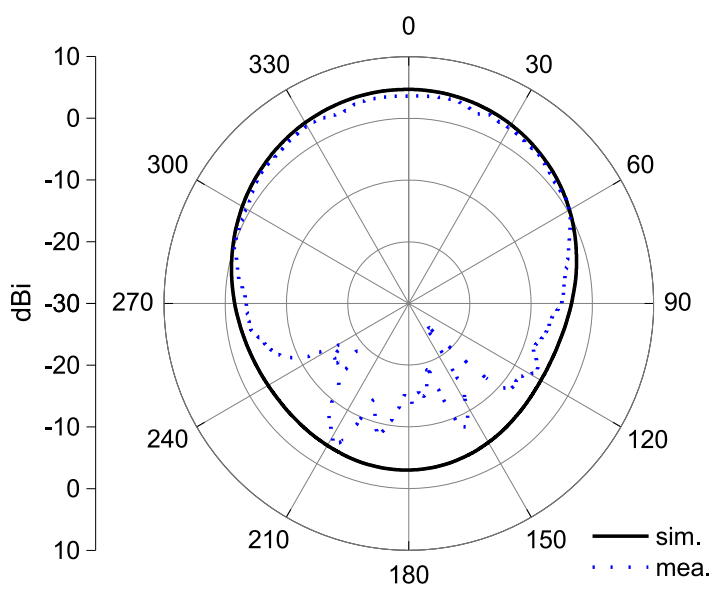

(b)

Fig. 4. The simulated and measured far-field radiation patterns of (a) DNG and ENG ZOR antennas (E-plane) [16] and (b) MNG ZOR antenna (E-plane) [17].

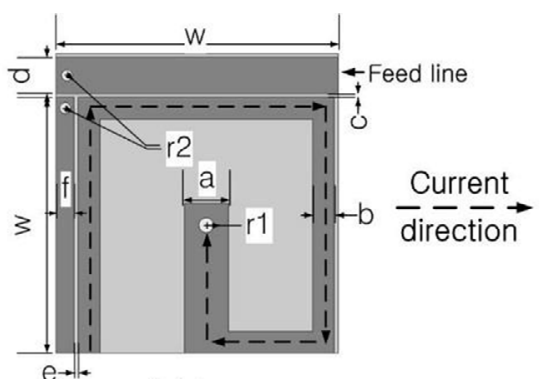

(a) top

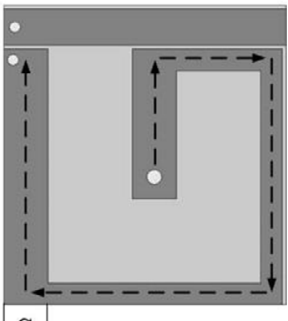

(b) bottom

Fig. 5. Geometry and surface current direction for MZR antenna with the same current path $(a=1.2 \mathrm{~mm}, b=0.6 \mathrm{~mm}, c=0.1 \mathrm{~mm}$, $d=1 \mathrm{~mm}, \quad e=0.1 \mathrm{~mm}, \quad f=0.5 \mathrm{~mm}, g=1.2 \mathrm{~mm}, \quad w=7.6 \mathrm{~mm}$, $r_{1}=0.15 \mathrm{~mm}, r_{2}=0.2 \mathrm{~mm}$ ) [18].

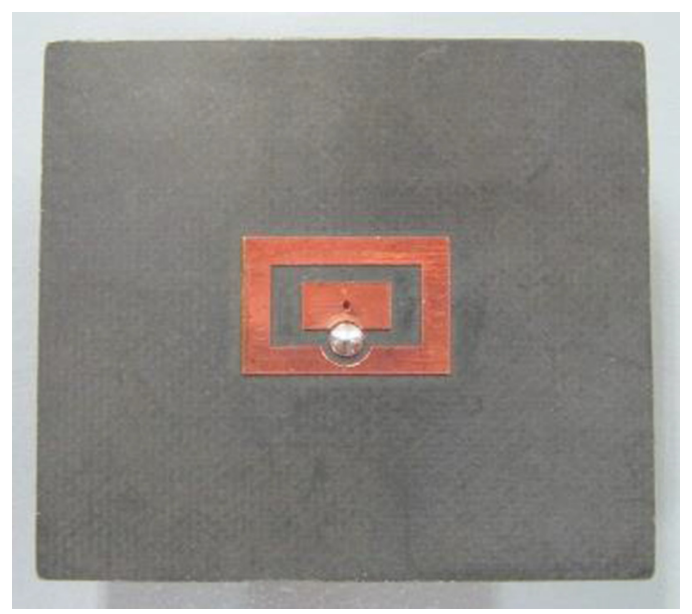

Fig. 7. Photograph of wide beamwidth antenna [20].

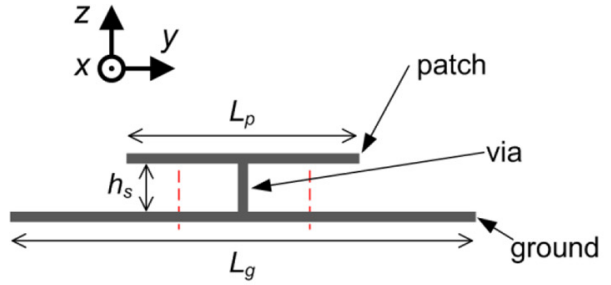

(a)

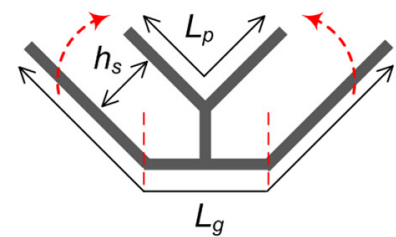

(b)

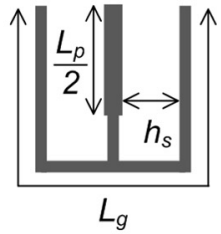

(c)

Fig. 6. Folding procedure (side view) (a) conventional mushroom antenna, (b) transforming stage, and (c) folded mushroom antenna [19].

\section{ZOR antennas for practical applications}

\subsection{Efficient wireless power transfer (WPT)}

An MZR with an effective zero permeability is presented for efficient WPT using resonant inductive coupling (RIC) [23]. An N-cell MZR is modified to maintain a fixed size and resonance frequencies that are important design factors of
WPT using RIC because they are related to the magnetic coupling coefficient and Q-factor. The resonator has many resonant modes with the extraordinary phenomena of metamaterials such as an infinite wavelength wave and backward-wave propagation. The 2-cell MZR is designed as shown in Figure 10. Figure 11a shows a photograph of the measurement setup and the power transfer efficiency of 2-cell MZR is simulated and measured as shown in 


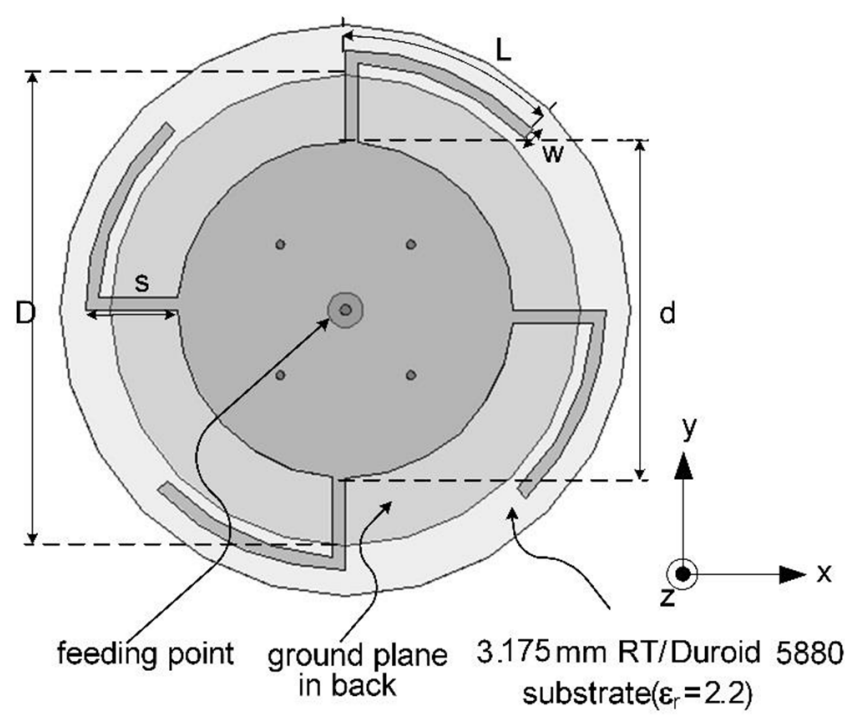

Fig. 8. Structure of the omnidirectional CP EZR antenna.

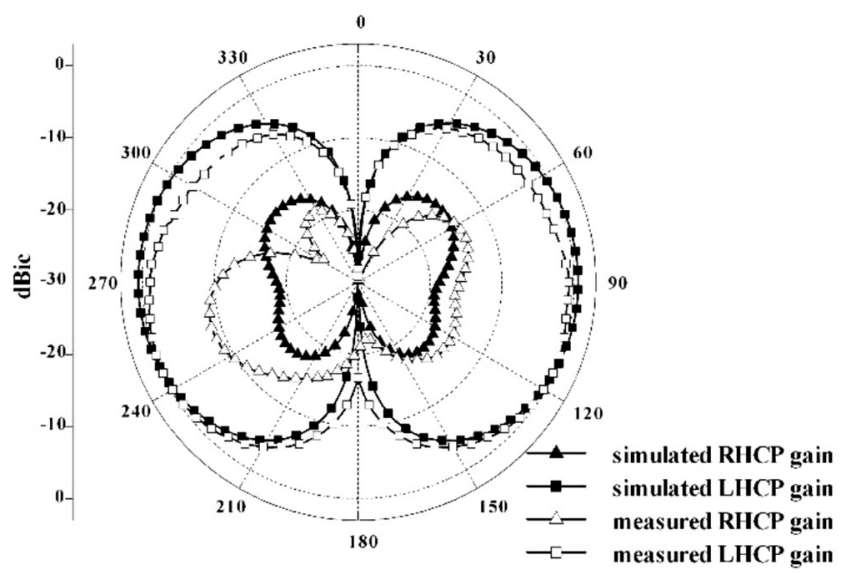

(a)
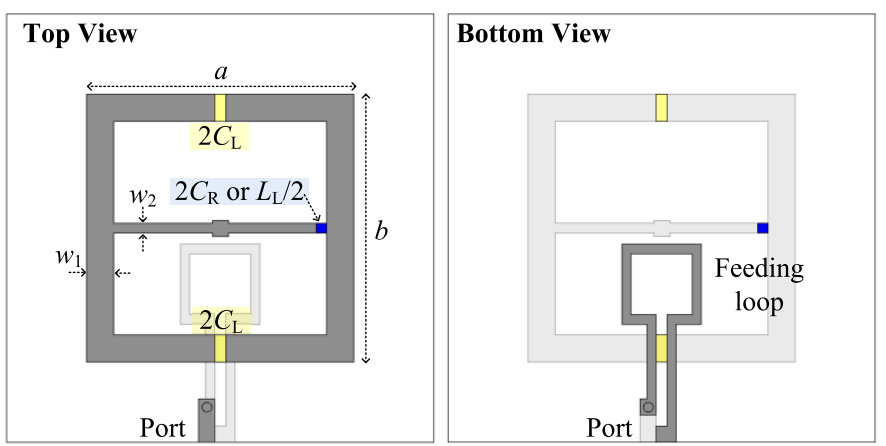

Fig. 10. Structure of the 2-cell MZR.

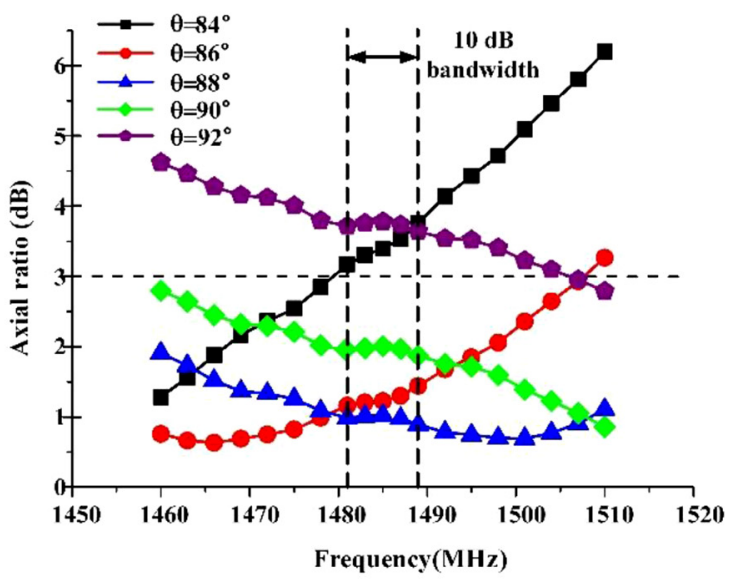

(b)

Fig. 9. (a) Simulated and measured radiation pattern $\left(x-z\right.$ plane). (b) Measured average axial ratio $\left(\theta=84^{\circ}, 86^{\circ}, 88^{\circ}, 90^{\circ}\right.$, and $\left.92^{\circ}\right)$ vs. frequency.

Figure 11b. To optimize the transfer efficiency of the WPT system using the MZR mode, which supports stronger coupling than the other modes, an equivalent circuit of muzero resonator is analyzed for a high Q-factor. To compare the characteristics of the proposed resonator and the other resonators of $[27,28]$, the size, frequency, distance, and WPT efficiencies are summarized in Table 1.

\subsection{Compact mobile antenna}

A compact penta-band dual ZOR antenna with band-stop filter is proposed for mobile applications [24]. The ZOR antenna is designed with modified mushroom-like structures having extended via on non-ground region to obtain good efficiency and broad bandwidth. Figure 12a and b shows the structure and photograph of the dual ZOR antenna with band-stop filter, respectively. This modified mushroom-like structure is confirmed as DNG transmis- sion line by full wave simulated dispersion relation. Moreover, a bended patch and a band-stop filter (BSF) are employed to increase efficiency and bandwidth, respectively. The length of each antenna is about $\lambda_{0} / 10$ at the resonant frequencies of $900 \mathrm{MHz}$ and $1800 \mathrm{MHz}$, respectively. The overall dimension of the antenna is $54.4 \mathrm{~mm}$ (length) $\times 4 \mathrm{~mm}$ (width) $\times 5 \mathrm{~mm}$ (height). The total efficiencies in low and high bands are measured more than $40 \%$ and 70\%, respectively, as shown in Figure 13. Table 2 shows comparison of performance between this paper and the other studies.

\subsection{Compact controlled reception pattern antenna (CRPA) array}

A compact CRPA array based on an MZR antenna is proposed for a global positioning system (GPS) [25]. The MZR antenna can be minimized by designing structure 


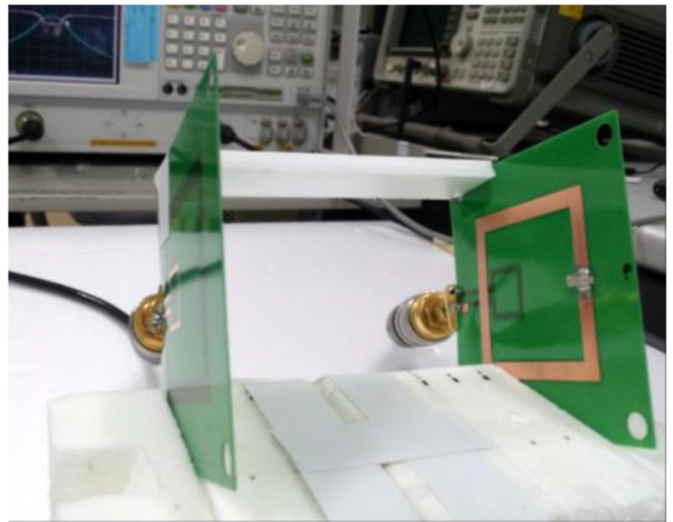

(a)

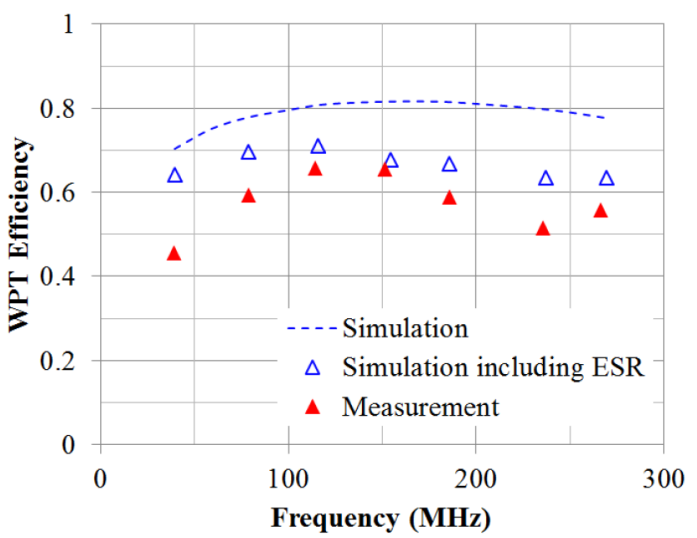

(b)

Fig. 11. (a) Photograph of the measurement setup and (b) simulated and measured WPT efficiency of two identical MZRs.

Table 1. Comparison of various resonators for WPT.

\begin{tabular}{|c|c|c|c|c|c|}
\hline WPT system Ref. & Size (length $\times$ length $\times$ height) & $\begin{array}{l}\text { Frequency } \\
(\mathrm{MHz})\end{array}$ & $\begin{array}{l}\text { Distance }(\mathrm{cm}) \\
(\text { length*2) }\end{array}$ & $\begin{array}{l}\text { Measured } \\
\text { WPT eff. (\%) }\end{array}$ & $\begin{array}{l}\text { Multi-band } \\
\text { application } \\
\text { including WPT }\end{array}$ \\
\hline$[27]$ & $\begin{array}{l}60 \mathrm{~cm} \times 60 \mathrm{~cm} \times 20 \mathrm{~cm} \\
\left(0.020 \lambda_{0} \times 0.020 \lambda_{0} \times 0.007 \lambda_{0}\right) \\
(\text { Helix type })\end{array}$ & 9.9 & 120 & 80 & No \\
\hline [28] & $\begin{array}{l}60 \mathrm{~cm} \times 60 \mathrm{~cm} \times 0.3 \mathrm{~cm}\left(0.015 \lambda_{0} \times\right. \\
\left.0.015 \lambda_{0} \times 0.00007 \lambda_{0}\right)(\text { Spiral type })\end{array}$ & 7.65 & 120 & 20 & No \\
\hline This paper & $\begin{array}{l}5 \mathrm{~cm} \times 5 \mathrm{~cm} \times 0.5 \mathrm{~cm}\left(0.0024 \lambda_{0} \times\right. \\
\left.0.0024 \lambda_{0} \times 0.0002 \lambda_{0}\right)\end{array}$ & 14.1 & 10 & 22 & Yes \\
\hline
\end{tabular}

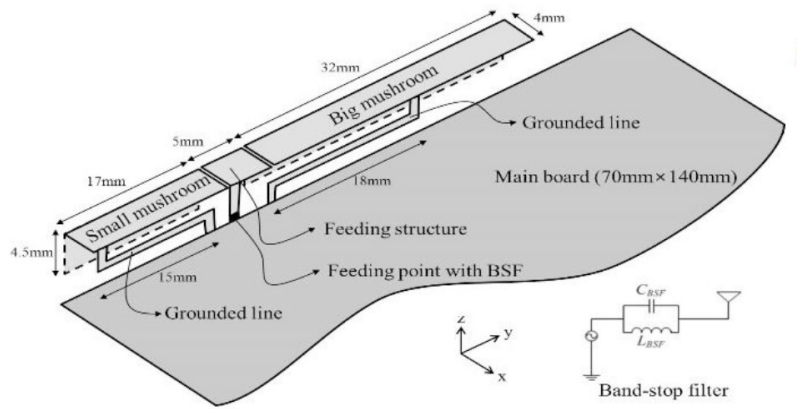

(a)

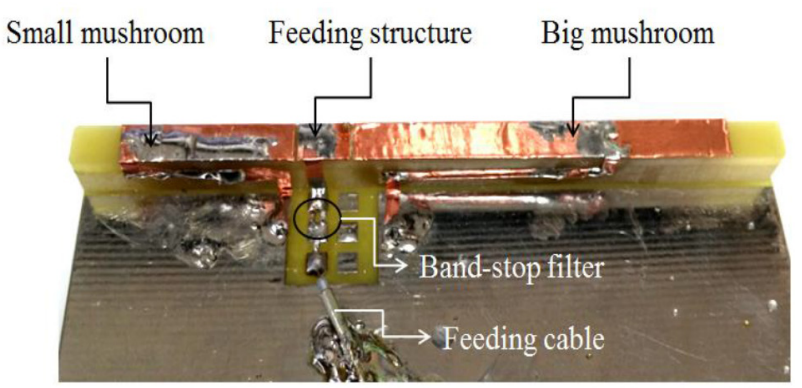

(b)

Fig. 12. (a) Structure and (b) photograph of the proposed dual ZOR antenna.

based on MNG transmission line. The MNG transmission line can be implemented by a gap structure for the series capacitance and a shorting via for a shortended boundary condition, as shown in Figure 14. The CRPA array, which operates in L1 $(1.57542 \mathrm{GHz})$ and L2 $(1.2276 \mathrm{GHz})$ bands, is designed as a cylinder with a diameter and a height of $127 \mathrm{~mm}$ ( 5 inches) and $20 \mathrm{~mm}$, respectively, and is composed of seven radiating elements. To design the compact CRPA array with high performance attributes such as an impedance matching (VSWR) value of less than 2 , an isolation between array elements $(<-12 \mathrm{~dB})$, an axial ratio $(<5 \mathrm{~dB})$, and a circular polarization $(\mathrm{CP})$ gain (>-1 dBic: L1 band and >-3 dBic: L2 band), we employ two orthogonal MZR antennas, a superstrate, and chip couplers. Figures 15 and 16 show photographs of the fabricated CRPA array and full-wave simulated and measured radiation pattern, respectively. The measured results show that all 7 ports of the CRPA array have good performances and the proposed array can be one of solutions for a compact CRPA array. 

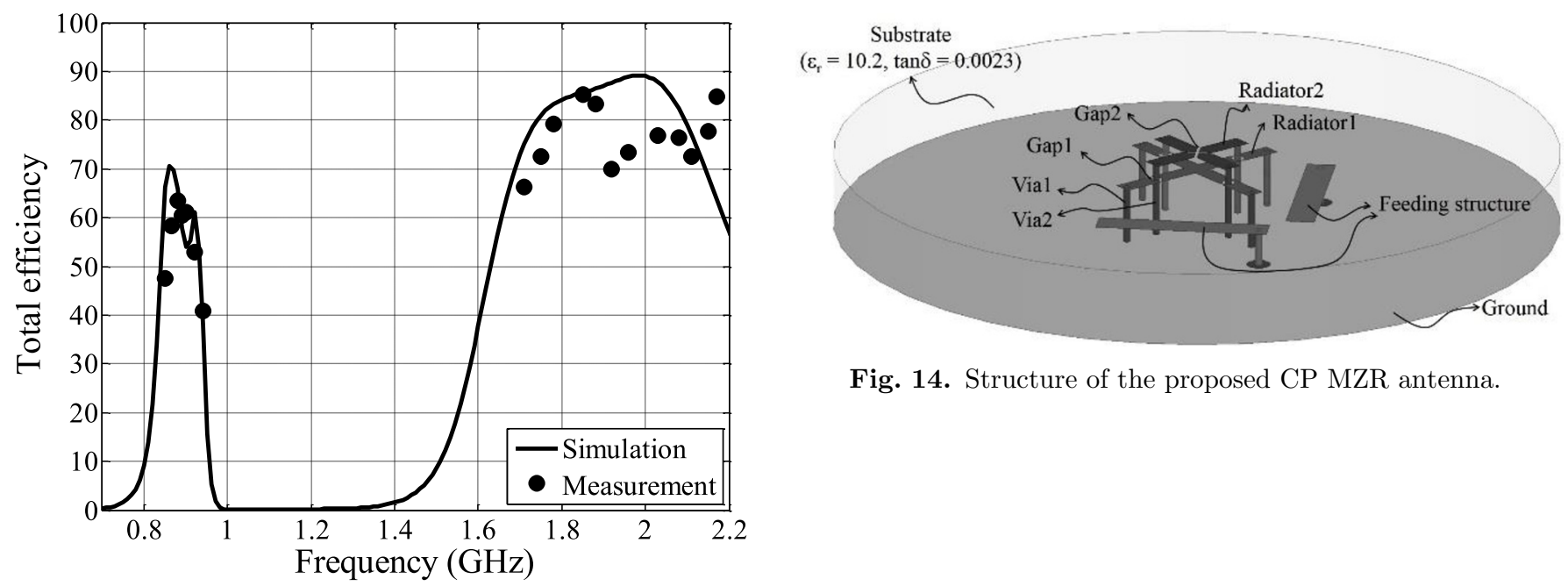

Fig. 14. Structure of the proposed CP MZR antenna.

Fig. 13. Simulated and measured total efficiency of dual ZOR antenna with BSF.

Table 2. Comparison of various mobile antenna.

\begin{tabular}{llll}
\hline Mobile antenna Ref. & Volume $($ length $\times$ width $\times$ height $)$ & Bands & Efficiency \\
\hline$[29]$ & $2400 \mathrm{~mm}^{3}(60 \mathrm{~mm} \times 4 \mathrm{~mm} \times 10 \mathrm{~mm})$ & Penta-band & $>60 \%$ (Low-band) $>60 \%$ (High-band) \\
{$[30]$} & $2200 \mathrm{~mm}^{3}(40 \mathrm{~mm} \times 11 \mathrm{~mm} \times 5 \mathrm{~mm})$ & Penta-band & $>50 \%$ (Low-band) $>60 \%$ (High-band) \\
This paper & $1088 \mathrm{~mm}^{3}(54.4 \mathrm{~mm} \times 4 \mathrm{~mm} \times 5 \mathrm{~mm})$ & Penta-band & $>40 \%$ (Low-band) $>70 \%$ (High-band) \\
\hline
\end{tabular}

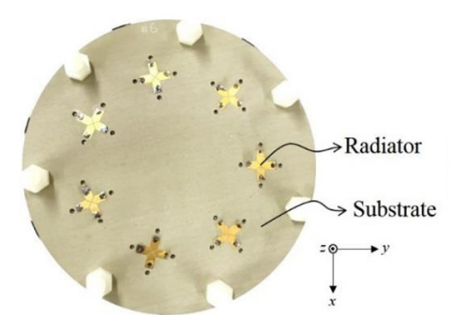

(a)

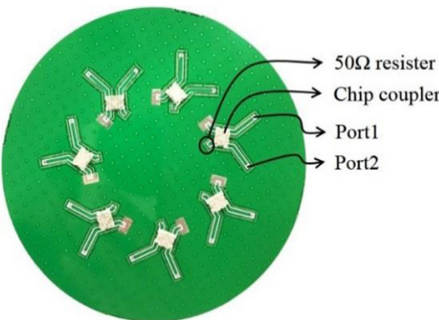

(b)

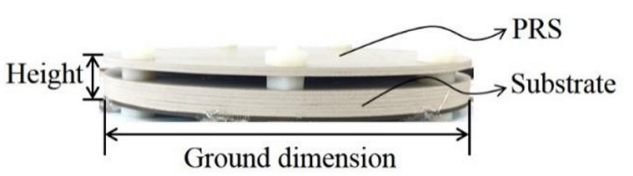

(c)

Fig. 15. Photographs of the fabricated CRPA array. (a) Top view before mounting superstrate, (b) bottom view, and (c) side view.

\subsection{Wide angle scanning CP array}

A meta-structured circular polarized array antenna for wide scan angle is proposed and designed. To widen the scanning angle of array antennas, this research investigates unit antenna beamwidth and the coupling effects between array elements, both of which directly affect the steering performance. As a result, the optimal array distance, the mode configuration, and the antenna structure are described. By using the features of the miniaturized MZR antenna in Figure 17, it is possible to design the antenna at optimum antenna spacing for wide beamwidth. In addition, by modifying via position and gap configuration of the antenna, it is possible to optimize the mode configuration for optimal isolation. Finally, the $3 \mathrm{~dB}$ steerable angle of $66^{\circ}$ is successfully demonstrated using a $1 \times 8 \mathrm{MZR} \mathrm{CP}$ antenna array without any additional decoupling structure, as shown in Figure 18. Figure 19 shows the simulated and measured beam patterns at a scan angle of $0^{\circ}, 22^{\circ}, 44^{\circ}$, and $66^{\circ}$.

\section{Conclusion}

Four kinds of meta-structured (DPS, DNG, ENG, and $\mathrm{MNG}$ ) transmission lines are classified, and the theoretical propagation constants and ZOR frequencies of the transmission lines are reviewed in this paper. From the theory, we have presented the DNG ZOR, EZR, and MZR antennas and the various deepening research such as electrically small, wideband, broad beamwidth, and CP, is conducted. Moreover, because the ZOR antennas have a good performance in the various applications, these types of antenna seem to be alternative to the conventional antenna. 

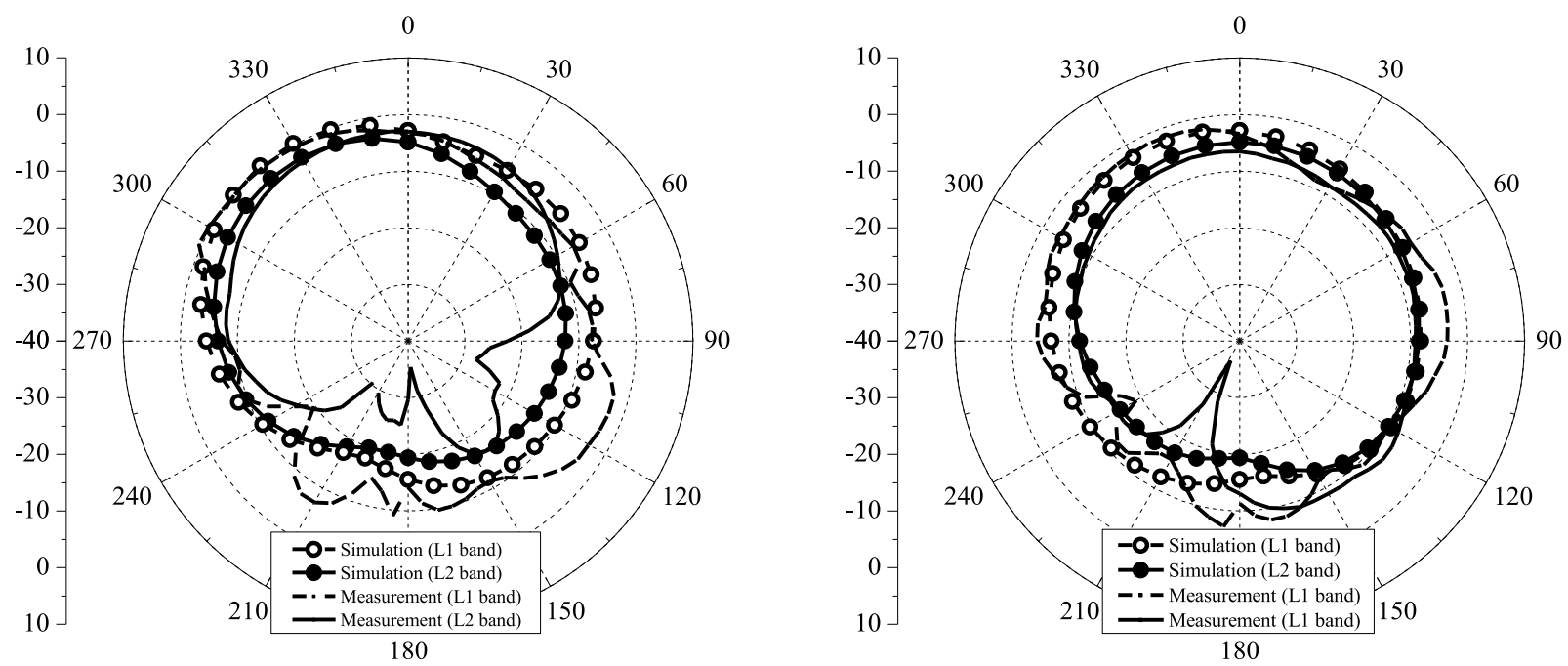

Fig. 16. Full-wave simulated and measured far-field radiation pattern (a) $y-z$ plane and (b) $x-z$ plane.

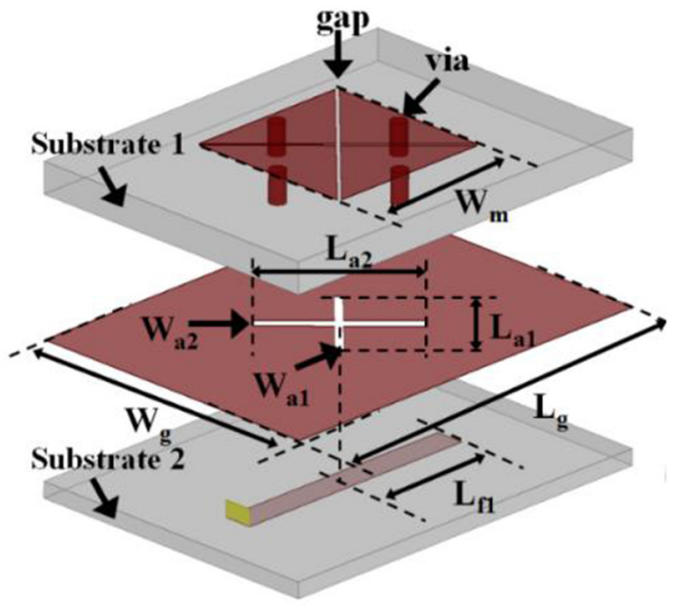

Fig. 17. Structure of proposed MZR CP antenna.

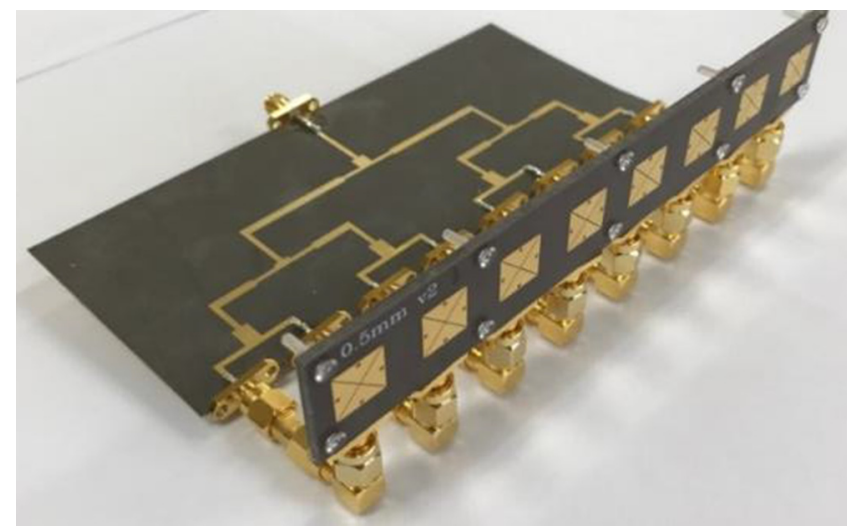

Fig. 18. Photograph of the fabricated $1 \times 8$ MZR array with phase delay lines for the steering angle of $0^{\circ}$.

This research was supported by Basic Science Research Program through the National Research Foundation of Korea (NRF) funded by the Ministry of Education (No. 2015R1A6A1A03031833) and MSIP (Ministry of Science, ICT and Future Planning), Korea, under the ITRC (Information

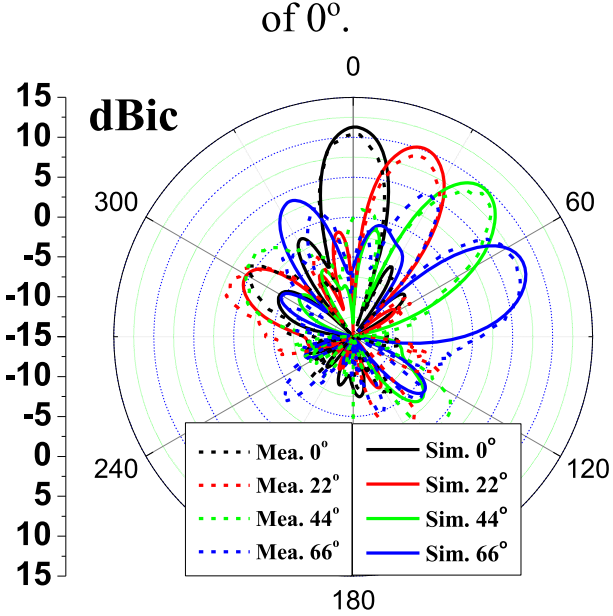

Fig. 19. Simulated and measured radiation patterns of $1 \times 8$ array antenna at various steering angles.

Technology Research Center) support program (IITP-2017-20160-00291) supervised by the IITP (Institute for Information \& communications Technology Promotion).

\section{References}

1. V.G. Veselago, The electrodynamics of substances with simultaneously negative values of $\varepsilon$ and $\mu$, Sov. Phys. Uspekhi 10, 509-514 (1968)

2. R.A. Shelby, D.R. Smith, S. Schultz, Experimental verification of a negative index refraction, Science 292, 77-79 (2001)

3. C. Caloz, T. Itoh, Transmission line approach of left-handed $(\mathrm{LH})$ materials and microstrip implementation of an artificial LH transmission line, IEEE Trans. Antennas Propag. 52, 1159-1166 (2004)

4. G.V. Eleftheriades, A.K. Iyer, P.C. Kremer, Planar negative refractive index media using periodically L-C loaded transmission lines, IEEE Trans. Microwave Theory Tech. 50, 2702-2712 (2002) 
5. C. Caloz, T. Itoh, A novel mixed conventional microstrip and composite right/left-handed backward-wave directional coupler with broadband and tight coupling characteristics, IEEE Microw. Wireless Comp. Lett. 14, 31-33 (2004)

6. J.G. Lee, J.H. Lee, Parallel coupled bandstop filter using double negative coupled transmission line, IEEE Microw. Wireless Comp. Lett. 17, 283 (2007)

7. J.G. Lee, J.H. Lee, Backward-wave directional coupler with complete coupling and broadband using conventional microstrip and 1D mushroom structure, Microw. Opt. Technol. Lett. 48, 1293-1296 (2006)

8. H. Okabe, C. Caloz, T. Itoh, A compact enhanced-bandwidth hybrid ring using an artificial lumped-element left-handed transmission-line section, IEEE Trans. Microw. Theory Tech. 52, 798-804 (2004)

9. O.F. Siddiqui, A.S. Mohra, G.V. Eleftheriades, Quad-band power divider based on left-handed transmission lines, Electr. Lett. 46, 1441-1442 (2010)

10. M. Studniberg, G.V. Eleftheriades, A dual-band bandpass filter based on generalized negative-refractive-index transmission-lines, IEEE Microw. Wireless Comp. Lett. 19, 18-20 (2009)

11. T. Yang, P.L. Chi, T. Itoh, Compact quarter-wave resonator and its applications to miniaturized diplexer and triplexer, IEEE Trans. Microw. Theory and Tech. 59, 260-269 (2011)

12. M. Gil, J. Bonache, J. Garcia, J. Martel, F. Martin, Composite right/left-handed metamaterial transmission lines based on complementary split-rings resonators and their applications to very wideband and compact filter design, IEEE Trans. Microw. Theory Tech. 55, 1296-1304 (2007)

13. A. Sanada, C. Caloz, T. Itoh, Novel zeroth-order resonance in composite right/left-handed transmission line resonators, Proc. Asia-Pacific Microw. Conf. 3, 1588-1591 (2003)

14. A. Sanada, M. Kimura, I. Awai, C. Caloz, T. Itoh, A planar zeroth-order resonator antenna using a left-handed transmission line, Proc. Eur. Microw. Conf. 3, 1341-1344 (2004)

15. J.G. Lee, J.H. Lee, Zeroth order resonance loop antenna, IEEE Trans. Antennas Propag. 55, 994-997 (2007)

16. J.H. Park, Y.H. Ryu, J.G. Lee, J.H. Lee, Epsilon negative zeroth-order resonator antenna, IEEE Trans. Antennas Propag. 55, 3710-3712 (2007)

17. J.H. Park, Y.H. Ryu, J.H. Lee, Mu-zero resonance antenna, IEEE Trans. Antennas Propag. 58, 1865-1875 (2010)
18. S.W. Lee, J.H. Lee, Electrically small MNG ZOR antenna with multi-layered conductor, IEEE Microw. Wireless Comp. Lett. 9, 724-727 (2010)

19. S.T. Ko, J.H. Lee, Wideband folded mushroom zeroth-order resonance antenna, IET Microw. Antennas Propag. 7, 79-84 (2013)

20. S.T. Ko, J.H. Lee, Hybrid zeroth-order resonance patch antenna with broad E-plane beamwidth, IEEE Trans. Antennas Propag. 61, 19-25 (2013)

21. B.C. Park, J.H. Lee, Omnidirectional circularly polarized antenna utilizing zeroth-order resonance of epsilon negative transmission line, IEEE Trans. Antennas and Propag. 59, 2717-2720 (2011)

22. B.C. Park, J.H. Lee, Dual-band omnidirectional circularly polarized antenna using zeroth- and first-order modes, IEEE Microw. Wireless Comp. Lett. 11, 407-410 (2012)

23. J.H. Park, B.C. Park, Y.H. Ryu, E.S. Park, J.H. Lee, Modified mu-zero resonator for efficient wireless power transfer, IET Microw. Antennas Propag. 8, 912-920 (2014)

24. J.G. Lee, D.J. Kim, J.H. Lee, Compact penta-band dual ZOR antenna for mobile applications, Int. J. Antennas Propag. 2016, 6461805 (2016)

25. J.G. Lee, T.S. Kwon, B.H. Choi, J.H. Lee, Compact controlled reception pattern antenna array based on muzero resonance antenna, IEICE Trans. Commun. E101-B, 1427-1433 (2018)

26. C.H. Lee, J.H. Lee, Wide angle scanning circular polarized meta-structured antenna array, IEICE Trans. Commun. E101-B, 2017-2023 (2018)

27. A. Kurs, A. Karalis, R. Moffatt, J.D. Joannopoulos, P. Fisher, M. Soljacic, Wireless power transfer via strongly coupled magnetic resonances, Science 317, 83-86 (2007)

28. A.P. Sample, D.T. Meyer, J.R. Smith, Analysis, experimental results, and range adaption of magnetically coupled resonators for wireless power transfer, IEEE Trans. Ind. Electr. 58, 544-554 (2011)

29. K. Wong, C. Huang, Printed loop antenna with a perpendicular feed for penta-band mobile phone application, IEEE Trans. Antennas Propag. 56, 2138-2141 (2008)

30. C. Peng, I. Chen, C. Hung, S. Shen, C. Chien, C. Tseng, Bandwidth enhancement of internal antenna by using reactive loading for penta-band mobile handset application, IEEE Trans. Antennas Propag. 59, 1728-1733 (2011) 